\title{
The auditory brain-stem response to complex sounds: a potential biomarker for guiding treatment of psychosis
}

\author{
Melissa A. Tarasenko, ${ }^{1,2}$, Neal R. Swerdlow ${ }^{2}$, Scott Makeig ${ }^{3}$, David L. Braff ${ }^{1,2}$ and Gregory A. Light ${ }^{1,2 *}$ \\ ${ }^{\prime}$ VISN-22 Mental IIIness, Research, Education and Clinical Center (MIRECC), VA San Diego Healthcare System, La Jolla, CA, USA \\ ${ }^{2}$ Department of Psychiatry, University of California San Diego, La Jolla, CA, USA \\ ${ }^{3}$ Swartz Center for Computational Neuroscience, Institute for Neural Computation, University of California San Diego, La Jolla, CA, USA
}

\section{Edited by:}

Judith M. Ford, Yale University School of Medicine, USA

Reviewed by:

Nina Kraus, Northwestern University, USA

Assen Veniaminov Jablensky, The University of Western Australia, Australia

\section{${ }^{*}$ Correspondence:}

Gregory A. Light, Department of Psychiatry, University of California San Diego, 9500 Gilman Drive,

La Jolla, CA 92093-0804, USA

e-mail: glight@ucsd.edu
Cognitive deficits limit psychosocial functioning in schizophrenia. For many patients, cognitive remediation approaches have yielded encouraging results. Nevertheless, therapeutic response is variable, and outcome studies consistently identify individuals who respond minimally to these interventions. Biomarkers that can assist in identifying patients likely to benefit from particular forms of cognitive remediation are needed. Here, we describe an event-related potential (ERP) biomarker - the auditory brain-stem response (ABR) to complex sounds (CABR) - that appears to be particularly well-suited for predicting response to at least one form of cognitive remediation that targets auditory information processing. Uniquely, the cABR quantifies the fidelity of sound encoded at the level of the brainstem and midbrain. This ERP biomarker has revealed auditory processing abnormalities in various neurodevelopmental disorders, correlates with functioning across several cognitive domains, and appears to be responsive to targeted auditory training. We present preliminary CABR data from 18 schizophrenia patients and propose further investigation of this biomarker for predicting and tracking response to cognitive interventions.

Keywords: auditory brain-stem response, biomarkers, cognitive remediation, complex auditory brain-stem response, EEG, psychosis, schizophrenia
Cognitive impairment is a core feature of schizophrenia that is associated with psychosocial functioning deficits [e.g., Ref. (1, 2)]. Neural network models of cognitive dysfunction in psychosis implicate a distributed neural architecture that includes "higher" cortical regions specialized for integrative cognitive operations as well as neural substrates of lower-level perceptual processing [e.g., Ref. $(3,4)]$. Consistent with these models, auditory perceptual deficits have been shown to contribute significantly to impaired cognition (5). Despite the fundamental role of subcortical structures in auditory processing and cognition, neurophysiological characterization of subcortical functioning is largely underrepresented in the schizophrenia literature. The auditory brainstem and midbrain, in particular, include a group of structures that support crucial functions in the representation of auditory information: the cochlear nuclei, superior olivary complex, lateral lemniscus, inferior and superior colliculi, and the auditory thalamus. These structures are sensitive to the subtle cues required for perception of pitch, timing, amplitude and localization of sounds, and they share reciprocal feedback with higher cortical regions through dense ascending and descending pathways (6). This subcortical network has an extremely well-established neurophysiological biomarker of functioning: the ABR. In fact, the ABR is among the most widely used clinical EEG measures with a variety of validated applications including newborn hearing screenings, auditory threshold estimation, intraoperative monitoring of auditory system function, detecting auditory nerve and brain-stem lesions, assessing for the presence of demyelinating conditions, detecting brain death, and determining coma type and recovery prognosis (7).
Despite abundant evidence of auditory system dysfunction in schizophrenia, surprisingly few studies have actually examined ABRs in this population, and results therein have been equivocal. Some studies have found normal ABRs $(8,9)$ while others have detected abnormal or even missing responses (1014). Interestingly, abnormal brain-stem activity has been associated with the presence of auditory hallucinations $(13,15)$, a hallmark symptom experienced by most schizophrenia patients at some point over the course of illness (16). More thorough assessment of brain-stem function in schizophrenia is, therefore, warranted.

One particularly promising subcortical target for future investigation is the inferior colliculus (IC) of the midbrain, a key auditory processing structure and convergence site for auditory pathways ascending from brainstem to cortex (6). The IC is crucial for maintaining fidelity of neural signals generated at the very earliest stages of auditory perception [i.e., in the cochlear nucleus and auditory nerve (17)]. Along with the nearby lateral lemniscus, IC exhibits the ability to phase-lock up to $1,000 \mathrm{~Hz}$ concordant with the acoustic properties of relatively complex sinusoidal sounds (18-22). Extant neurophysiologic research on brainstem and midbrain function in schizophrenia has only examined ABRs evoked in response to simple click stimuli (click ABRs), which lack the complexity necessary to evoke this "frequency-following" response. Speech and other more acoustically complex sounds, however, do elicit such a response and could thus provide a more sensitive assessment of brainstem and midbrain neurophysiology in schizophrenia. 


\section{COMPLEX AUDITORY BRAIN-STEM RESPONSE}

The complex auditory brain-stem response (cABR), shown in Figure 1, is an event-related potential (ERP) with an onset approximately $6 \mathrm{~ms}$ after presentation of an acoustically complex sound. Its peak amplitudes and latencies correspond with the acoustic properties of its evoking stimulus $(23,24)$, and it is thought to provide an objective index of the brainstem and midbrain's representation of complex sounds [cf. Ref. (25)]. Although cABRs can be evoked in response to various types of complex stimuli, here, we primarily focus on those evoked by consonant-vowel speech sounds (e.g., /da/or/mi/). As illustrated in Figure 1, portions of the $\mathrm{cABR}$ uniquely correspond to speech stimulus parameters namely, the stop consonant onset, consonant-to-vowel formant transition, frequency-following response to the vowel sound, and offset of voicing. Neural representation of stimulus pitch, timing, and timber can be derived from the waveform, with high correlation between $\mathrm{CABR}$ and stimulus suggesting accurate encoding of sound (25-27). Abnormal cABRs can be characterized by a number of features including small peak amplitudes and long peak latencies relative to the stimulus sound wave (i.e., small stimulus-to-response correlation), low signal-to-noise ratio, weak phase-locking activity, and response variability over time. Such abnormalities have been demonstrated in a number of clinical conditions, including specific language impairment, dyslexia, and autism $(25,28,29)$. Older adults have also demonstrated abnormalities in comparison with their younger counterparts (30, 31). Perhaps the most compelling argument for investigating the cABR in schizophrenia comes from Russo et al., who showed that children with autism exhibit abnormal cABRs in the context of intact click-ABRs (29), suggesting that speech and perhaps other complex sounds may offer improved sensitivity over traditional click-ABR measures for detecting brainstem and midbrain dysfunction.

\section{UTILITY OF THE CABR AS A TREATMENT BIOMARKER IN PSYCHOSIS}

Numerous higher level sequelae of cABR abnormalities have been identified, with robust relationships demonstrated between cABRs and reading abilities (32), phonological processing (33-35), perception of speech in background noise (36), language learning (37), auditory selective attention (38), auditory learning (39), auditory working memory (40), and executive functioning (38). Given that deficits in several of these domains are also commonly present in patients with psychotic illnesses, the cABR may serve as a sensitive index of neural dysfunction occurring within the earliest stages of auditory processing that subsequently cascades "forward" to affect the engagement of higher cortical networks known to underlie cognitive deficits in schizophrenia, or that may otherwise reflect pathology common to both brainstem and higher brain areas.

Among the more intriguing findings from cABR research thus far is that, contrary to conventional beliefs about the "fixedness" of subcortical structures and their relatively passive role in auditory perception $(41,42)$, brain-stem activity can seemingly be modified as a function of experience with sound. Musicians, presumed to be "auditory experts" due to their extensive training in pitch discrimination and other auditory skills, exhibit stronger correspondence between cABRs and speech stimuli than do non-musicians, and cABR pitch-tracking accuracy is positively correlated with years of musical training (43-45). Language experience can also influence

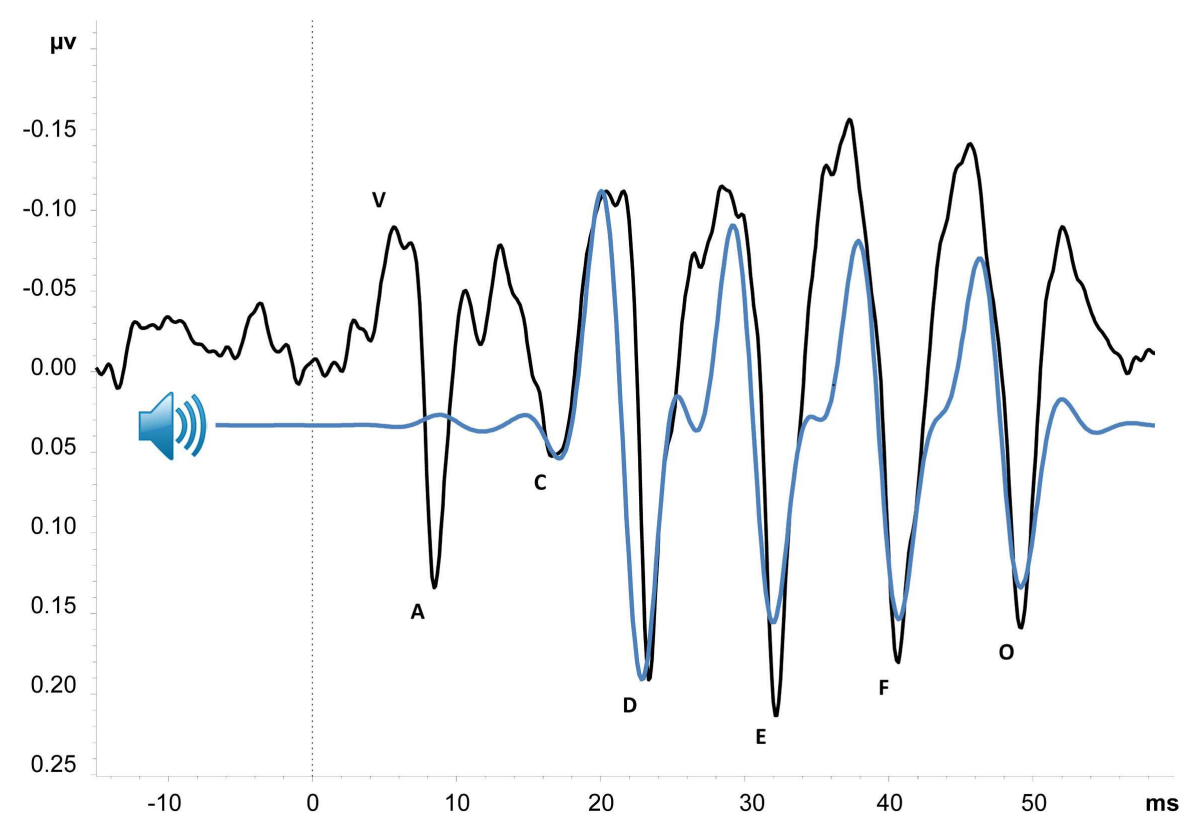

FIGURE 1 | Grand average cABR from 18 schizophrenia patients. Black line is average $C A B R$ from $C z$; peaks are named in accordance with convention [e.g. (27)]. Blue line represents "da" stimulus sound wave, $200 \mathrm{~Hz}$ low-pass filtered to better illustrate similarities between CABR and stimulus. Sound wave has also been shifted forward approximately six milliseconds to account for transmission time from cochlea to brainstem. Peaks $\mathrm{V}-\mathrm{A}, \mathrm{C}$, and $\mathrm{O}$ are transient features of the $\mathrm{CABR}$, corresponding with stop consonant onset, consonant-vowel formant transition, and offset of voicing, respectively. Peaks $D, E$, and $F$ comprise the sustained frequency-following response (FFR) of the vowel sound (Tarasenko et al., in preparation). 
brain-stem activity, as evidenced by greater fidelity of cABRs in tonal language speakers (46) and bilinguals (38). Notably, the benefits of auditory experience on acoustic processing can also be reaped through targeted short-term interventions, with increased cABR fidelity having been found following training programs containing an auditory discrimination training component (47-50). In fact, Skoe et al. (51) demonstrated transient cABR modification after only 15 min of auditory training, leading the authors to characterize the brainstem as a "barometer of rapid auditory learning."

The malleability of cABRs in response to even a brief course of auditory training is particularly relevant to current treatment development efforts in psychosis. Recently, cognitive training programs have capitalized on existing knowledge of sensory disruption in psychosis by incorporating auditory frequency discrimination exercises that are designed to place implicit and increasing demands on basic auditory perception. Targeted cognitive training (TCT) is one such approach, aiming to improve cognition in part by "sharpening" the fidelity of auditory processing. Data increasingly suggest that targeted "tuning" of underlying neural systems is indeed beneficial for facilitating cognitive recovery in schizophrenia, with patients demonstrating large improvements in auditory-dependent domains of verbal learning and memory and verbal working memory that generalize to enhanced global cognition $(d=0.86-0.89)$ following $50 \mathrm{~h}$ of TCT (52). Despite evidence of TCT's efficacy at the group level, however, individual responses to the training are highly variable, with some patients showing virtually no cognitive improvement even after an extended course of $100 \mathrm{~h}$ of training (53). There is, thus, a need to identify ERP biomarkers that are sensitive to neurophysiological changes occurring early in TCT and may predict response to a full "dose" of this and other resource-intensive cognitive interventions.

The identification of biomarkers has been a high priority for psychiatry research, due to mounting evidence of overlapping neural networks that underlie multiple psychiatric illnesses, calling into question the validity of traditional symptom-based diagnostic categories $(54,55)$. Biomarkers that provide direct assays of the neural circuits underlying clinical phenomena may allow for more precise diagnosis and reliable estimation of benefit from interventions targeting clinically relevant neural circuits (56). Although substantial progress has been made in validating a number of viable candidates (57-62), a "gold standard" ERP biomarker of cognitive dysfunction in psychosis has not yet been established. Scalp ERP measures that currently permeate this literature (e.g., amplitudes and latencies of peaks P50, N1, MMN, P300, etc.) reflect relatively high-level brain responses to sound stimuli and have been shown to sum potentials from multiple cortical source areas $(3,4,63,64)$. Peak cABR measures, in contrast, provide an objective probe of sound representation and have little intra-individual variation in the absence of a systematic auditory training regimen (65); their ability to reliably quantify the degree of disruption present in the auditory signal may thus proffer a unique advantage for accurately diagnosing psychotic disorders and predicting likelihood of benefit from TCT and/or other cognitive, pharmacologic, or combined interventions. Reported adaptations in brain-stem responses early in the course of auditory training [e.g., after only 15 min (51)] could suggest both engagement of the targeted neural system and a capability for neural plasticity required to benefit more broadly from further training.

Importantly, the ERP paradigms used to study cABRs are ideal for inclusion in a biomarker test battery, as they are typically short in duration (approximately $15 \mathrm{~min}$ ) and require little attention or effort from participants, who typically engage in a distracting visual task during auditory stimulus presentation [see in Ref. (27) for further discussion of task parameters]. Preliminary data, shown in Figure 1, suggest that this type of task is indeed feasible to administer and is well tolerated by schizophrenia patients. A measure of cABR functioning could therefore be easily added to existing batteries to better inform diagnosis and guide subsequent treatment.

\section{CONCLUSION}

Neurocognitive deficits commonly found in schizophrenia patients may in part reflect dysfunction in lower-level auditory processing mechanisms that have conventionally been studied with click-ABRs and other neurophysiological biomarkers [e.g., Ref. $(54,56,57,59,60,66,67)]$. The cABR provides an objective, multidimensional measure of sound encoding that is abnormal in some neurodevelopmental disorders, and these deficits are associated with impaired performance across several higher-order cognitive domains. This measure may serve as a sensitive biomarker that predicts or corresponds to therapeutic response to auditory-based cognitive training interventions for schizophrenia.

\section{ACKNOWLEDGMENTS}

The authors thank Erika Skoe for methodological consultation and Marlena Pela for assisting with data collection. This paper was supported by the VISN-22 Mental Illness, Research, Education, and Clinical Center, the Brain and Behavior Research Foundation (NARSAD), the Veterans Medical Research Foundation, and the National Institutes of Mental Health (UL1TR000100, MH42228, MH065571, MH094151, MH093453, MH094320, MH081944, MH59803), and by a gift to Scott Makeig from The Swartz Foundation (Old Field, NY, USA).

\section{REFERENCES}

1. Green MF. What are the functional consequences of neurocognitive deficits in schizophrenia? Am J Psychiatry (1996) 153:321-30.

2. Green MF, Kern RS, Braff DL, Mintz J. Neurocognitive deficits and functional outcome in schizophrenia: are we measuring the "right stuff"? Schizophr Bull (2000) 26:119-36. doi:10.1093/oxfordjournals.schbul.a033430

3. Rissling AJ, Miyakoshi M, Sugar CA, Braff DL, Makeig S, Light GA. Cortical substrates and functional correlates of auditory deviance processing deficits in schizophrenia. Neuroimage (in press).

4. Takahashi H, Rissling AJ, Pascual-Marqui R, Kirihara K, Pela M, Sprock J, et al. Neural substrates of normal and impaired preattentive sensory discrimination in large cohorts of nonpsychiatric subjects and schizophrenia patients as indexed by MMN and P3a change detection responses. Neuroimage (2012) 66C:594-603. doi:10.1016/j.neuroimage.2012.09.074

5. Kawakubo Y, Kasai K, Kudo N, Rogers MA, Nakagome K, Itoh K, et al. Phonetic mismatch negativity predicts verbal memory deficits in schizophrenia. Neuroreport (2006) 17:1043-6. doi:10.1097/01.wnr.0000221828.10846.ba

6. Middlebrooks JC. Auditory system: central pathways. In: Squire LR, editor. Encyclopedia of Neuroscience. Oxford: Academic Press (2009). p. 745-52.

7. Petrova LD. Brainstem auditory evoked potentials. Am J Electroneurodiagnostic Technol (2009) 49:317-32. 
8. Brecher M, Begleiter H. Brain stem auditory evoked potentials in unmedicated schizophrenia patients. Biol Psychiatry (1985) 20:199-202. doi:10.1016/00063223(85)90082-4

9. Pfefferbaum A, Horvath TB, Roth WT, Tinklenberg JR, Kopell BS. Auditory brain stem and cortical evoked potentials in schizophrenia. Biol Psychiatry (1980) 2:209-23.

10. Hayashida Y, Mitani Y, Hosomi H, Amemiya M, Mifune K, Tomita S. Auditory brainstem responses in relation to the clinical symptoms of schizophrenia. Biol Psychiatry (1986) 21:177-88. doi:10.1016/0006-3223(86)90145-9

11. Igata M, Ohta M, Hayashida Y, Abe K. Missing peaks in auditory brainstem responses and negative symptoms in schizophrenia. Psychiatry Clin Neurosci (1994) 48:571-8. doi:10.1111/j.1440-1819.1994.tb03016.x

12. Källstrand J, Nehlstedt SF, Sköld ML, Nielzén S. Lateral asymmetry and reduced forward masking effect in early brainstem auditory evoked responses in schizophrenia. Psychiatry Res (2012) 196:188-93. doi:10.1016/j.psychres.2011. 08.024

13. Lindström L, Klockhoff I, Svedberg A, Bergstrom K. Abnormal auditory brainstem responses in hallucinating schizophrenic patients. Br J Psychiatry (1987) 151:9-14. doi:10.1192/bjp.151.1.9

14. Lindström LH, Wieselgren IM, Klockhoff I, Svedberg A. Relationship between abnormal brainstem auditory-evoked potentials and subnormal CSF levels of HVA and 5-HIAA in first-episode schizophrenic patients. Biol Psychiatry (1990) 28:435-42. doi:10.1016/0006-3223(90)90411-T

15. Shergill SS, Brammer MJ, Williams SC, Murray RM, McGuire PK. Mapping auditory hallucinations in schizophrenia using functional magnetic resonance imaging. Arch Gen Psychiatry (2000) 57:1033-8. doi:10.1001/archpsyc.57.11.1033

16. Mueser KT, Bellack AS, Brady EU. Hallucinations in schizophrenia. Acta Psychiatr Scand (1990) 82:26-9. doi:10.1111/j.1600-0447.1990.tb01350.x

17. Dhar S, Abel R, Hornickel J, Nicol T, Skoe E, Zhao W, et al. Exploring the relationship between physiological measures of cochlear and brainstem function. Neurophysiol Clin (2009) 120:959-66. doi:10.1016/j.clinph.2009.02.172

18. Hoormann J, Falkenstein M, Hohnsbein J, Blanke L. The human frequencyfollowing response (FFR): normal variability and relation to the click-evoked brainstem response. Hear Res (1992) 59(2):179-88. doi:10.1016/0378-5955(92) 90114-3

19. Marsh JT, Worden FG, Smith JC. Auditory frequency-following response: neural or artifact? Science (1970) 169:1222-3. doi:10.1126/science.169.3951.1222

20. Moushegian G, Rupert AL, Stillman RD. Laboratory note. Scalp-recorded early responses in man to frequencies in the speech range. Electroencephalogr Clin Neurophysiol (1973) 35:665-7. doi:10.1016/0013-4694(73)90223-X

21. Smith JC, Marsh JT, Brown WS. Far-field recorded frequency-following responses: evidence for the locus of brainstem sources. Electroencephalogr Clin Neurophysiol (1975) 39:465-72. doi:10.1016/0013-4694(75)90047-4

22. Worden FG, Marsh JT. Frequency-following (microphonic-like) neural responses evoked by sound. Electroencephalogr Clin Neurophysiol (1968) 25:42-52. doi:10.1016/0013-4694(68)90085-0

23. Banai K, Abrams D, Kraus N. Sensory-based learning disability: insights for brainstem processing of speech sounds. Int J Audiol (2007) 46:524-32. doi:10.1080/14992020701383035

24. Russo N, Nicol T, Musacchia G, Kraus N. Brainstem responses to speech syllables. Neurophysiol Clin (2004) 115:2021-30. doi:10.1016/j.clinph.2004.04.003

25. Kraus N, Chandrasekaran B. Music training for the development of auditory skills. Nat Rev Neurosci (2010) 11:599-605. doi:10.1038/nrn2882

26. Hornickel J, Kraus N. Objective biological measures for the assessment and management of auditory processing disorder. Curr Pediatr Rev (2011) 7:252-61. doi:10.2174/157339611796548438

27. Skoe E, Kraus N. Auditory brainstem response to complex sounds: a tutorial. Ear Hear (2010) 31:302-24. doi:10.1097/AUD.0b013e3181cdb272

28. Basu M, Krishnan A, Weber-Fox C. Brainstem correlates of temporal auditory processing in children with specific language impairment. Dev Sci (2010) 13:77-91. doi:10.1111/j.1467-7687.2009.00849.x

29. Russo NM, Skoe E, Trommer B, Nicol T, Zecker S, Bradlow A, et al. Deficient brainstem encoding of pitch in children with autism spectrum disorders. Neurophysiol Clin (2008) 119:1720-31. doi:10.1016/j.clinph.2008.01.108

30. Clinard CG, Tremblay KL, Krishnan AR. Aging alters the perception and physiological representation of frequency: evidence from human frequencyfollowing response recordings. Hear Res (2010) 264:48-55. doi:10.1016/j.heares. 2009.11.010
31. Clinard CG, Tremblay KL. Aging degrades the neural encoding of simple and complex sounds in the human brainstem. J Am Acad Audiol (2013) 24:590-9. doi:10.3766/jaaa.24.7.7

32. Banai K, Hornickel JM, Skoe E, Nicol T, Zecker S, Kraus N. Reading and subcortical auditory function. Cereb Cortex (2009) 19:2699-707. doi:10.1093/cercor/ bhp024

33. Hornickel J, Skoe E, Nicol T, Zecker S, Kraus N. Subcortical differentiation of voiced stop consonants: relationships to reading and speech in noise perception. Proc Natl Acad Sci U S A (2009) 106:13022-7. doi:10.1073/pnas. 0901123106

34. Hornickel J, Chandrasekaran B, Zecker S, Kraus N. Auditory brainstem measures predict reading and speech-in-noise perception in school-aged children. Behav Brain Res (2011) 216:597-605. doi:10.1016/j.bbr.2010.08.051

35. Hornickel J, Kraus N. Unstable representation of sound: a biological marker of dyslexia. J Neurosci (2013) 33:3500-4. doi:10.1523/JNEUROSCI.4205-12. 2013

36. Anderson S, Kraus N. Sensory-cognitive interaction in the neural encoding of speech in noise: a review. J Am Acad Audiol (2010) 21:575-85. doi:10.3766/jaaa. 21.9.3

37. Chandrasekaran B, Kraus N, Wong PCM. Human inferior colliculus activity relates to individual differences in spoken language learning. J Neurophysiol (2012) 107:1325-36. doi:10.1152/jn.00923.2011

38. Krizman J, Marian V, Shook A, Skoe E, Kraus N. Subcortical encoding of sound is enhanced in bilinguals and relates to executive function advantages. Proc Natl Acad Sci U S A (2012) 109:7877-81. doi:10.1073/pnas.1201575109

39. Skoe E, Chandrasekaran B, Spitzer ER, Wong PC, Kraus N. Human brainstem plasticity: the interaction of stimulus probability and auditory learning. Neurobiol Learn Mem (2014) 109:82-93. doi:10.1016/j.nlm.2013.11.011

40. Strait DL, Parbery-Clark A, Hittner E, Kraus N. Musical training during early childhood enhances the neural encoding of speech in noise. Brain Lang (2012) 123:191-201. doi:10.1016/j.bandl.2012.09.001

41. Hickok G, Poeppel D. The cortical organization of speech processing. Nat Rev Neurosci (2007) 8:393-402. doi:10.1038/nrn2113

42. Rauschecker JP, Scott SK. Maps and streams in the auditory cortex: nonhuman primates illuminate human speech processing. Nat Neurosci (2009) 12:718-24. doi:10.1038/nn.2331

43. Musacchia G, Sams M, Skoe E, Kraus N. Musicians have enhanced subcortical auditory and audiovisual processing of speech and music. Proc Natl Acad Sci U $S$ A (2007) 104:15894-8. doi:10.1073/pnas.0701498104

44. Parbery-Clark A, Skoe E, Kraus N. Musical experience limits the degradative effects of background noise on the neural processing of sound. J Neurosci (2009) 29:14100-7. doi:10.1523/JNEUROSCI.3256-09.2009

45. Wong PCM, Skoe E, Russo NM, Dees T, Kraus N. Musical experience shapes human brainstem encoding of linguistic pitch patterns. Nat Neurosci (2007) 10:420-2. doi:10.1038/nn1872

46. Krishnan A, Gandour JT, Bidelman GM. The effects of tone language experience on pitch processing in the brainstem. J Neurolinguistics (2010) 23:81-95. doi:10.1016/j.jneuroling.2009.09.001

47. Anderson S, White-Schwoch T, Parbery-Clark A, Kraus N. Reversal of age-related neural timing delays with training. Proc Natl Acad Sci U S A (2013) 110:4357-62. doi:10.1073/pnas.1213555110

48. Carcagno S, Plack CJ. Subcortical plasticity following perceptual learning in a pitch discrimination task. J Assoc Res Otolaryngol (2011) 12:89-100. doi:10.1007/s10162-010-0236-1

49. Skoe E, Kraus N. Hearing it again and again: on-line subcortical plasticity in humans. PLoS One (2010) 5:e13645. doi:10.1371/journal.pone.0013645

50. Song JH, Skoe E, Wong PC, Kraus N. Plasticity in the adult human auditory brainstem following short-term linguistic training. J Cog Neurosci (2008) 20:1892-902. doi:10.1162/jocn.2008.20131

51. Skoe E, Krizman J, Spitzer E, Kraus N. The auditory brainstem is a barometer of rapid auditory learning. Neuroscience (2013) 243:104-14. doi:10.1016/j. neuroscience.2013.03.009

52. Fisher M, Holland C, Merzenich MM, Vinogradov S. Using neuroplasticitybased auditory training to improve verbal memory in schizophrenia. Am J Psychiatry (2009) 166:805-11. doi:10.1176/appi.ajp.2009.08050757

53. Fisher M, Holland C, Subramaniam K, Vinogradov S. Neuroplasticity-based cognitive training in schizophrenia: an interim report on the effects 6 months later. Schizophr Bull (2010) 36:869-79. doi:10.1093/schbul/sbn170 
54. Light GA, Swerdlow NR. Neurophysiological biomarkers informing the clinical neuroscience of schizophrenia: mismatch negativity and prepulse inhibition of startle. Curr Top Behav Neurosci (2014). doi:10.1007/7854_2014_316

55. Swerdlow NR. Are we studying and treating schizophrenia correctly? Schizophr Res (2011) 130:1-10. doi:10.1016/j.schres.2011.05.004

56. Perez VB, Swerdlow NR, Braff DL, Natanen R, Light GA. Using biomarkers to inform diagnosis, guide treatments and track response to interventions in psychotic illnesses. Biomark Med (2014) 8:9-14. doi:10.2217/bmm.13.133

57. Braff DL, Light GA. Preattentional and attentional cognitive deficits as targets for treating schizophrenia. Psychopharmacology (2004) 174:75-85. doi:10.1007/ s00213-004-1848-0

58. Javitt DC, Spencer KM, Thaker GK, Winterer G, Hajos M. Neurophysiological biomarkers for drug development in schizophrenia. Nat Rev Drug Discov (2008) 7:68-83. doi:10.1038/nrd2463

59. Light GA, Hsu JL, Hsieh MH, Meyer-Gomes K, Sprock J, Swerdlow NR, et al. Gamma band EEG oscillations reveal neural network cortical coherence dysfunction in schizophrenia patients. Biol Psychiatry (2006) 60:1231-40. doi:10.1016/j.biopsych.2006.03.055

60. Light GA, Naatanen R. Mismatch negativity is a breakthrough biomarker for understanding and treating psychotic disorders. Proc Natl Acad Sci U S A (2013) 110:15175-6. doi:10.1073/pnas.1313287110

61. Light GA, Swerdlow NR, Thomas ML, Calkins ME, Green MF, Greenwood TA, et al. Validation of mismatch negativity and P3a for use in multi-site studies of schizophrenia: characterization of demographic, clinical, cognitive, and functional correlates in COGS-2. Schizophr Res (in press).

62. Turetsky BI, Dress EM, Braff DL, Calkins ME, Green MF, Greenwood TA, et al. The utility of $\mathrm{P} 300$ as a schizophrenia endophenotype and predictive biomarker: clinical and socio-demographic modulators in COGS-2. Schizophr Res (in press).

63. Makeig S, Westerfield M, Jung T-P, Enghoff S, Townsend J, Courchesne E, et al. Dynamic brain sources of visual evoked responses. Science (2002) 295:690-4. doi:10.1126/science. 1066168
64. Makeig S, Delorme A, Westerfield M, Jung T-P, Townsend J, Courchesne E, et al. Electroencephalographic brain dynamics following manually responded visual targets. PLoS Biol (2004) 2:e-176. doi:10.1371/journal.pbio.0020176

65. Kraus N, Nicol T. The cognitive auditory system: the role of learning in shaping the biology of the auditory system. In: Fay R, Popper A, editors. Perspectives on Auditory Research. Springer Handbook of Auditory Research. Heiderlberg: Springer-Verlag (2014). p. 299-319.

66. Javitt DC. When doors of perception close: bottom-up models of disrupted cognition in schizophrenia. Ann Rev Clin Psychol (2009) 5:249-75. doi:10.1146/ annurev.clinpsy.032408.153502

67. Kirihara K, Rissling AJ, Swerdlow NR, Braff DL, Light GA. Hierarchical organization of gamma and theta oscillatory dynamics in schizophrenia. Biol Psychiatry (2012) 71:873-80. doi:10.1016/j.biopsych.2012.01.016

Conflict of Interest Statement: Dr. Swerdlow is a consultant for Genco Sciences, Inc. Dr. Light has served as a consultant for Astellas, Forum, and NeuroVerse for matters unrelated to this study. Drs. Tarasenko, Makeig, and Braff report no biomedical financial interests or potential conflicts of interest.

Received: 30 July 2014; paper pending published: 02 September 2014; accepted: 25 September 2014; published online: 13 October 2014.

Citation: Tarasenko MA, Swerdlow NR, Makeig S, Braff DL and Light GA (2014)

The auditory brain-stem response to complex sounds: a potential biomarker for guiding treatment of psychosis. Front. Psychiatry 5:142. doi: 10.3389/fpsyt.2014.00142

This article was submitted to Schizophrenia, a section of the journal Frontiers in Psychiatry.

Copyright (c) 2014 Tarasenko, Swerdlow, Makeig, Braff and Light. This is an openaccess article distributed under the terms of the Creative Commons Attribution License (CC BY). The use, distribution or reproduction in other forums is permitted, provided the original author(s) or licensor are credited and that the original publication in this journal is cited, in accordance with accepted academic practice. No use, distribution or reproduction is permitted which does not comply with these terms. 